\title{
Phase-map measurements by interferometry with sinusoidal phase modulation and four integrating buckets
}

\author{
Arnaud Dubois \\ Laboratoire d'Optique, Ecole Supérieure de Physique et Chimie Industrielles, Centre National de la Recherche \\ Scientifique, UPR A0005, 10 rue Vauquelin, F-75231 Paris Cedex 5, France
}

Received September 18, 2000; revised manuscript received January 5, 2001; accepted January 29, 2001

\begin{abstract}
Phase-shifting interferometry based on the integrating-bucket technique with sinusoidal phase modulation is studied theoretically and demonstrated experimentally to obtain phase maps from double-beam interferometers. The method uses four frames obtained by integration of the time-varying intensity in an interference pattern during the four quarters of the modulation period. An optimum sinusoidal phase modulation is found to minimize the effect of the additive noise. The absolute accuracy of the phase measurements is discussed. Possible applications of the method are demonstrated with two interference microscopes with which the phase modulation is achieved by sinusoidal oscillation of a mirror attached to a piezoelectric transducer and by sinusoidal birefringence modulation with a photoelastic modulator. In both experimental arrangements, phase images can be produced in real time at a rate of several hertz. Noise measurements are reported and compared with theory. (c) 2001 Optical Society of America

OCIS codes: $120.5050,120.3180,120.5060,180.3170$.
\end{abstract}

\section{INTRODUCTION}

The invention of phase-shifting interferometry (PSI) was a major breakthrough in the field of interferometry, providing a method to measure the optical phase to unprecedented accuracy. ${ }^{1-4}$ PSI has been performed with almost all types of interferometric imaging systems through use of different algorithms to extract a phase map from several intensity fringe patterns. ${ }^{1-4}$ For all algorithms, a discrete or continuous temporal phase shift is introduced. By measuring the intensity as the phase is shifted, the phase can be obtained. In the so-called phase-stepping technique, the phase is stepped by a known amount between each intensity measurement, whereas in the socalled integrating-bucket technique the intensity is integrated while the phase is being shifted. A large number of phase-stepping algorithms have been proposed. ${ }^{5-15}$ In the integrating-bucket technique, the phase is usually shifted linearly in a sawtoothlike manner, and several integrated intensity values (or "buckets") are recorded. ${ }^{16-18}$ PSI that uses sinusoidal phase modulation is much less usual. ${ }^{19,20}$ We study in this paper an algorithm with sinusoidal phase modulation and four integrating buckets, as already proposed in Ref. 21 . We first present the principle of the method to obtain a phase map from the sinusoidally phase-modulated interference signal. Using analytical calculations, we show how to optimize the modulation parameters in order to minimize the effect of the additive noise. The influence of deviation of these modulation parameters is analyzed. The accuracy of the method for phase measurements is discussed. The method was applied on several interferometer systems. We present in particular two interference microscopes with which the phase modulation is induced by sinusoidal oscillation at $50 \mathrm{~Hz}$ of a mirror attached to a piezoelectric transducer (PZT) and with a photoelastic birefringence modulator operating at $50 \mathrm{kHz}$. Noise measurements are reported and compared with theory.

\section{PRINCIPLE}

In the absence of noise, assuming monochromatic illumination, the photon flux or intensity (these quantities are proportional to each other, related by $h \nu$, $h$ being the Planck constant and $\nu$ being the photon frequency) produced by double-beam interferometers can be written at a designated point $(x, y)$ in the transverse plane as

$$
I(x, y)=\bar{I}(x, y)+A(x, y) \cos \phi(x, y),
$$

where $\bar{I}$ is the bias or average photon flux, $A$ is the amplitude of the interference fringes, and $\phi$ is the optical phase. We describe a method to extract the optical-phase map $\phi$ by introducing a sinusoidal phase modulation of amplitude $\psi$, phase $\theta$ and period $T=2 \pi / \omega$ (we shall see later how this can be achieved in practice). Because of this phase modulation, the photon flux (or intensity) varies with time as

$$
\begin{gathered}
I(x, y, t)=\bar{I}(x, y)+A(x, y) \cos [\phi(x, y) \\
+\psi \sin (\omega t+\theta)] .
\end{gathered}
$$

The time-varying signal $I(t)$ is integrated successively over the four quarters of the modulation period $T$ as represented schematically in Fig. 1. The integration is performed in a parallel manner by a two-dimensional detector array, such as a CCD. The charge storage period of the image sensor is then set to be one-quarter period of the sinusoidal phase modulation. Four images (four frames of interferogram) are thus recorded. These four 


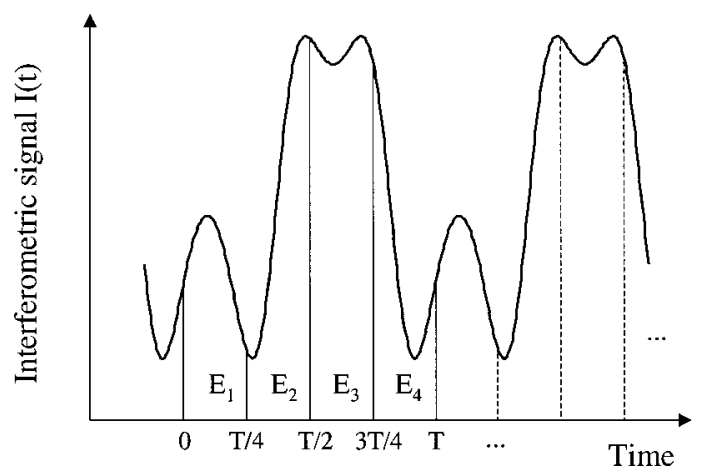

Fig. 1. Simulation of the interferometric signal $I(t)$ and how it is integrated over the four quarters of the modulation period $(\gamma=2 / 3, \phi=2, \psi=2.45$, and $\theta=0.98)$.

frames, obtained through integration of the time-varying signal during the four quarters of the modulation period $T$ (see Fig. 1), are

$$
E_{p}(x, y)=\int_{(p-1) T / 4}^{p T / 4} I(x, y, t) \mathrm{d} t, \quad p=1,2,3,4 .
$$

We disgard now the dependence on $x$ and $y$ to simplify the notations. To calculate the integral in Eq. (3), we write the signal $I(t)$ as a sum of Fourier components by using the Bessel functions of first kind $J_{n}$ :

$$
\begin{aligned}
I(t)=\bar{I} & +A J_{0}(\psi) \cos \phi+2 A \cos \phi \\
& \times \sum_{n=1}^{+\infty} J_{2 n}(\psi) \cos [2 n(\omega t+\theta)]-2 A \sin \phi \\
& \times \sum_{n=0}^{+\infty} J_{2 n+1}(\psi) \sin [(2 n+1)(\omega t+\theta)] .
\end{aligned}
$$

The integration of Eq. (3), with Eq. (4), gives

$$
\begin{aligned}
E_{p}= & (T / 4)\left(\bar{I}+A J_{0}(\psi) \cos \phi\right) \\
& +(T / \pi) A \cos \phi \sum_{n=1}^{+\infty} \frac{J_{2 n}(\psi)}{2 n}\{\sin [n p \pi+2 n \theta] \\
& -\sin [n(p-1) \pi+2 n \theta]\} \\
& -(T / \pi) A \sin \phi \sum_{n=0} \frac{J_{2 n+1}(\psi)}{2 n+1} \\
& \times\{\cos [(2 n+1)(p-1) \pi / 2+(2 n+1) \theta] \\
& -\cos [(2 n+1) p \pi / 2+(2 n+1) \theta]\} .
\end{aligned}
$$

Linear combinations of the four frames can be formed to have

$\Sigma_{s}=-E_{1}+E_{2}+E_{3}-E_{4}=(4 T / \pi) \Gamma_{s} A \sin \phi$,

$\Sigma_{c}=-E_{1}+E_{2}-E_{3}+E_{4}=(4 T / \pi) \Gamma_{c} A \cos \phi$,

where

$$
\begin{aligned}
\Gamma_{s} & =\sum_{n=0}^{+\infty}(-1)^{n} \frac{J_{2 n+1}(\psi)}{2 n+1} \sin [(2 n+1) \theta], \\
\Gamma_{c} & =\sum_{n=0}^{+\infty} \frac{J_{4 n+2}(\psi)}{2 n+1} \sin [2(2 n+1) \theta] .
\end{aligned}
$$

The phase map $\phi$ can then be obtained with the following equation:

$$
\tan \phi=\frac{\Gamma_{c}}{\Gamma_{s}} \frac{\Sigma_{s}}{\Sigma_{c}} .
$$

The calculation of the phase map $\phi$ involves the ratio of linear combinations of the four frames and depends on the amplitude $\psi$ and phase $\theta$ of the sinusoidal phase modulation through the parameters $\Gamma_{s}$ and $\Gamma_{c}$.

\section{OPTIMUM SINUSOIDAL PHASE MODULATION}

Which values should be given to the amplitude $\psi$ and phase $\theta$ of the sinusoidal phase modulation (see Eq. 2)? To answer this question, we studied the influence of the additive noise on the calculation of the optical phase $\phi$. Our approach is similar to the one presented in Ref. 21. Each of the four frames $E_{p}$ is assumed to have a Gaussian additive noise $n_{p}$ with the following properties (the angular brackets " \langle\rangle " denote the infinite-time average):

$$
\begin{aligned}
\left\langle n_{p}\right\rangle & =0 \quad p=1,2,3,4, \\
\left\langle n_{i} n_{j}\right\rangle & =\left\{\begin{array}{cc}
\sigma^{2}, & i=j \\
0, & i \neq j
\end{array}, \quad\left|n_{p}\right| \ll E_{p} .\right.
\end{aligned}
$$

Because of this noise, an error $\varepsilon$ appears in the calculation of the phase $\phi$. We propose to establish analytical expressions for the mean $\langle\varepsilon\rangle$ and the mean square $\left\langle\varepsilon^{2}\right\rangle$ of the error $\varepsilon$. In presence of the additive noise, the phase $\phi$ verifies the following equation:

$$
\eta=\tan (\phi+\varepsilon)=\frac{\Gamma_{c}}{\Gamma_{s}}\left(\frac{\Sigma_{s}+N_{s}}{\Sigma_{c}+N_{c}}\right)=\tan \phi \frac{1+N_{s} / \Sigma_{s}}{1+N_{c} / \Sigma_{c}}
$$

where

$$
\begin{aligned}
& N_{s}=-n_{1}+n_{2}+n_{3}-n_{4}, \\
& N_{c}=-n_{1}+n_{2}-n_{3}+n_{4} .
\end{aligned}
$$

Because the noise is assumed to be much weaker than the signal itself, the quantities $\eta$ and $\eta^{2}$ can be approximated as

$$
\begin{aligned}
& \eta \approx \tan \phi\left(1+\frac{N_{s}}{\Sigma_{s}}\right)\left(1-\frac{N_{c}}{\Sigma_{c}}+\frac{N_{c}^{2}}{\Sigma_{c}^{2}}\right), \\
& \eta^{2} \approx \tan ^{2} \phi\left(1+2 \frac{N_{s}}{\Sigma_{s}}+\frac{N_{s}^{2}}{\Sigma_{s}^{2}}\right) \\
& \times\left(1-2 \frac{N_{c}}{\Sigma_{c}}+3 \frac{N_{c}^{2}}{\Sigma_{c}^{2}}\right) .
\end{aligned}
$$

The mean values of $\eta$ and $\eta^{2}$ are

$$
\begin{aligned}
\langle\eta\rangle & =\tan \phi\left(1+\frac{4 \sigma^{2}}{\Sigma_{c}^{2}}\right), \\
\left\langle\eta^{2}\right\rangle & =\tan ^{2} \phi\left[1+4 \sigma^{2}\left(\frac{1}{\Sigma_{s}^{2}}+\frac{3}{\Sigma_{c}^{2}}\right)\right] .
\end{aligned}
$$


On the other hand, with a Taylor development at the second order,

$$
\eta=\tan (\phi+\varepsilon) \approx a_{0}+a_{1} \varepsilon+a_{2} \varepsilon^{2},
$$

where

$$
\begin{aligned}
& a_{0}=\tan \phi, \quad a_{1}=1+\tan ^{2} \phi, \\
& a_{2}=\tan \phi\left(1+\tan ^{2} \phi\right) .
\end{aligned}
$$

The mean values of $\eta$ and $\eta^{2}$ at the second order are

$$
\begin{aligned}
\langle\eta\rangle & =a_{0}+a_{1}\langle\varepsilon\rangle+a_{2}\left\langle\varepsilon^{2}\right\rangle, \\
\left\langle\eta^{2}\right\rangle & =a_{0}{ }^{2}+2_{0} a_{1}\langle\varepsilon\rangle+\left(a_{1}{ }^{2}+2 a_{0} a_{2}\right)\left\langle\varepsilon^{2}\right\rangle .
\end{aligned}
$$

Solving Eqs. (18) and (19) for $\langle\epsilon\rangle$ and $\left\langle\varepsilon^{2}\right\rangle$, we obtain

$$
\begin{aligned}
\langle\varepsilon\rangle= & \frac{1}{a_{1}^{3}}\left[-a_{0} a_{1}^{2}-a_{2} a_{0}^{2}+\left(a_{1}^{2}+2 a_{0} a_{2}\right)\langle\eta\rangle\right. \\
& \left.-a_{2}\left\langle\eta^{2}\right\rangle\right], \\
\left\langle\varepsilon^{2}\right\rangle= & \frac{1}{a_{1}^{2}}\left[a_{0}^{2}-2 a_{0}\langle\eta\rangle+\left\langle\eta^{2}\right\rangle\right] .
\end{aligned}
$$

Replacing the coefficients $a_{0}, a_{1}, a_{2}$ by their expressions [Eq. (17)], we have

$$
\begin{aligned}
\langle\varepsilon\rangle= & -\sin \phi \cos \phi-\sin ^{3} \phi \cos \phi+\cos ^{2} \phi\left(1+2 \sin ^{2} \phi\right) \\
& \times\langle\eta\rangle-\sin \phi \cos ^{3} \phi\left\langle\eta^{2}\right\rangle, \\
\left\langle\varepsilon^{2}\right\rangle= & \cos ^{4} \phi\left[\tan ^{2} \phi-2 \tan \phi\langle\eta\rangle+\left\langle\eta^{2}\right\rangle\right] .
\end{aligned}
$$

Substitution of Eqs. (14) and (15) into Eqs. (22) and (23) yields

$$
\begin{aligned}
\langle\varepsilon\rangle & =4 \sigma^{2} \sin \phi \cos \phi\left(\frac{\cos ^{2} \phi}{\Sigma_{c}^{2}}-\frac{\sin ^{2} \phi}{\Sigma_{s}^{2}}\right) \\
& =\frac{\sigma^{2} \pi^{2}}{4 A^{2} T^{2}} \sin \phi \cos \phi\left(\frac{1}{\Gamma_{c}{ }^{2}}-\frac{1}{\Gamma_{s}{ }^{2}}\right),
\end{aligned}
$$

$$
\begin{aligned}
\left\langle\varepsilon^{2}\right\rangle & =4 \sigma^{2} \sin ^{2} \phi \cos ^{2} \phi\left(\frac{1}{\Sigma_{c}{ }^{2}}+\frac{1}{\Sigma_{s}{ }^{2}}\right) \\
& =\frac{\sigma^{2} \pi^{2}}{4 T^{2} A^{2}}\left(\frac{\sin ^{2} \phi}{\Gamma_{c}{ }^{2}}+\frac{\cos ^{2} \phi}{\Gamma_{s}{ }^{2}}\right) .
\end{aligned}
$$

According to Eq. (24), it turns out that the mean phase error $\langle\varepsilon\rangle$ is zero for any value of the phase $\phi$ only when $\Gamma_{c}=\Gamma_{s}$. This first condition should therefore be fullfield. Then, according to Eq. (25), the mean square phase error $\left\langle\varepsilon^{2}\right\rangle$ is minimum when $\Gamma_{c}$ and $\Gamma_{s}$ are maximum. Therefore the optimum values for $\psi$ and $\theta$ are those for which $\Gamma_{c}$ and $\Gamma_{s}$ are equal and maximum. We have calculated numerically that this situation happens when $\psi=2.45$ and $\theta=0.98$, as can be seen graphically in Fig. 2. These results are in agreement with those reported in Ref. 21. With these optimum phase-modulation parameters, we have $\Gamma_{c}=\Gamma_{s}=\Gamma=0.405$. Then, the mean phase error is zero and its standard deviation is minimum, given by

$$
\sqrt{\left\langle\varepsilon^{2}\right\rangle_{\min }}=\frac{\sigma \pi}{2 T A \Gamma} .
$$

The phase $\phi$ is then obtained with the simplified formula

$$
\tan \phi=\frac{\Sigma_{s}}{\Sigma_{c}}=\frac{E_{1}-E_{2}-E_{3}+E_{4}}{E_{1}-E_{2}+E_{3}-E_{4}}
$$

We shall see in Section 7 how the optimal phasemodulation parameters $\psi=2.45$ and $\theta=0.98$ can be adjusted in practice.

\section{RESIDUAL NOISES}

The amplitude and phase of the sinusoidal-phase modulation being set to their optimum values, the phase map is obtained with a cancelled mean error and a minimized rms error corresponding to the residual noise. We propose in this section to evaluate the residual noise of the

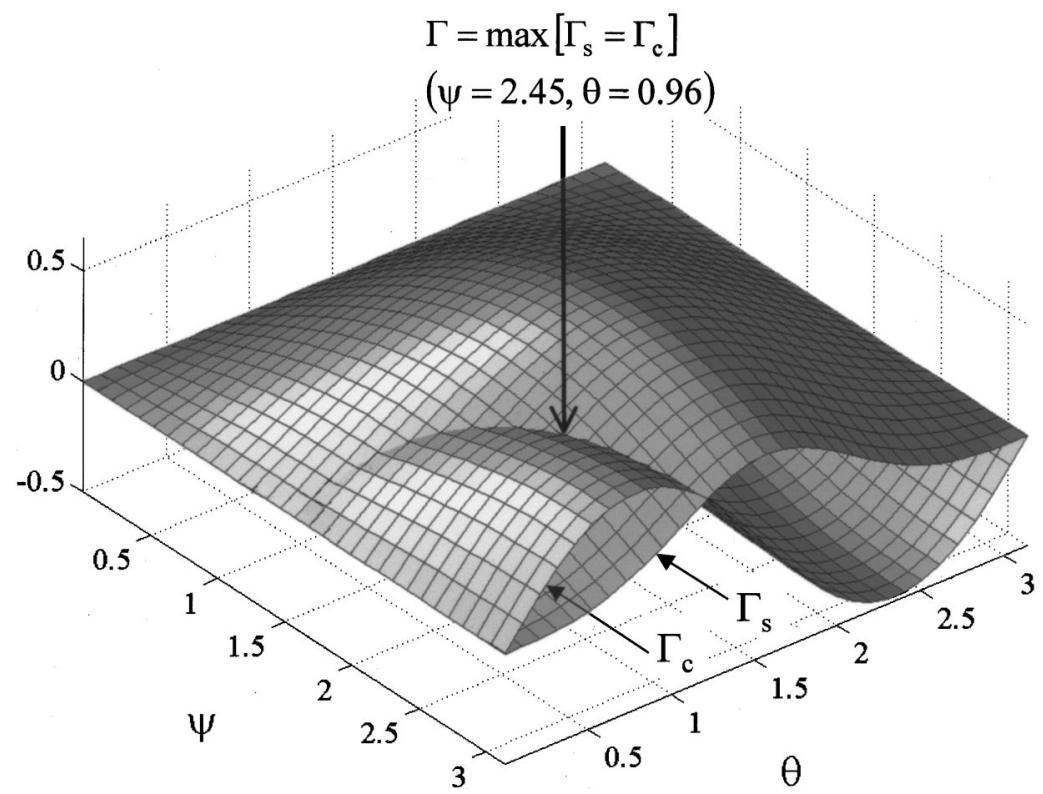

Fig. 2. Graphic representation of $\Gamma_{c}$ and $\Gamma_{s}: z=\Gamma_{c}(\psi, \theta)$ and $z=\Gamma_{s}(\psi, \theta)$ for $0<\psi, \theta<\pi$. The intersection of the two maps $z=\Gamma_{c}(\psi, \theta)=\Gamma_{s}(\psi, \theta)$ reaches its maximum value $\Gamma=0.405$ for $\psi=2.45$ and $\theta=0.98$. 
calculated phase by considering the measurements affected by two noises with different origins, the shot noise, and the noise resulting from the quantization error.

\section{A. Shot Noise}

Ultimately, the measurements are limited by the fundamental photon noise. Ordinarily this noise is Poisson distributed. The variance of the number of collected photons is equal to the number of collected photons itself. The $N$-collected photons generate $\zeta N$ charges stored on each pixel of the detector. $\zeta$ is the quantum efficiency of the detector assumed to be equal to 1 . If we consider a phase map with values uniformly distributed between 0 and $2 \pi$, the average number of charges stored by each pixel for each of the four frames is then [see Eq. (5)]

$$
N=\left\langle E_{p}\right\rangle=\bar{I} T / 4 \text {. }
$$

The average shot noise on each pixel is characterized by the standard deviation

$$
\sigma=\sqrt{N}
$$

Introducing the fringe contrast

$$
\gamma=A / \bar{I}
$$

we obtain from Eq. (26) the standard deviation of the phase (or rms phase noise) as

$$
\sqrt{\left\langle\varepsilon^{2}\right\rangle}=\pi /(8 \Gamma \gamma \sqrt{N}) \approx 1 /(\gamma \sqrt{N}) .
$$

If the shot noise is the predominate noise source, the noise present in the calculated phase map depends only on the contrast of the fringes $\gamma$ and the number of photoelectrons $N$ detected by each elementary detector in average for each frame. The full well-charge storage capability of the image sensor is thus an important criterion. Given that the phase is related to the optical path difference $2 z$ by

$$
\phi=4 \pi z / \lambda,
$$

the rms noise for distance measurements (in air), normalized by the optical wavelength, is given by

$$
\Delta z / \lambda=1 /(32 \Gamma \gamma \sqrt{N}) \approx 0.08 /(\gamma \sqrt{N}) .
$$

If we consider a CCD detector with an average wellcharge storage per pixel of $N=100,000$ and a fringe contrast of $\gamma=0.7$, the rms phase noise is equal to $\sqrt{\left\langle\varepsilon^{2}\right\rangle}$ $=4.410^{-3}$, which corresponds to $\sim \lambda / 2800$. Using a 840 -nm light source, the rms noise $\Delta z$ would be $\sim 300 \mathrm{pm}$.

\section{Table 1. Phase Noise Resulting from the Quantization Error ${ }^{a}$}

\begin{tabular}{lccc}
\hline Quantization levels (L) & 256 & 512 & 1024 \\
$\quad$ Mean filled levels & 152 & 306 & 612 \\
rms phase noise (mrad) & 2.6 & 1.3 & 0.6 \\
$\quad$ [from Eq. (36)] & & & \\
$\begin{array}{l}\text { rms noise } \Delta z \\
\text { [from Eq.(37)] }\end{array}$ & $\lambda / 4300$ & $\lambda / 8600$ & \\
\hline
\end{tabular}

\footnotetext{
${ }^{a}$ The fringe contrast is assumed to be $\gamma=0.7$, and the phase map is assumed to have values uniformly distributed between 0 and $2 \pi$. The mean filled levels are found numerically, assuming that the whole dynamic of the CCD is used in the pixels where the intensity is maximum.
}

For a given detector, the effect of the shot noise can be reduced by accumulating several measurements.

\section{B. Effect of Quantization Error}

The quantization error comes from truncating an infinitely precise intensity measurement to its nearestinteger representation. According to Ref. 22, this error may be considered as a uniformly distributed random variable ranging in value from $-1 / 2$ to $+1 / 2$, with a standard deviation

$$
\sigma=1 / \sqrt{12}
$$

The average number $L$ of quantization levels actually filled on average on each pixel of the detector for each frame is

$$
L=\left\langle E_{p}\right\rangle=\bar{I} T / 4 .
$$

According to Eq. (26), the standard deviation of the phase fluctuation (or rms phase noise) due to quantization is

$$
\sqrt{\left\langle\varepsilon^{2}\right\rangle}=\pi /(16 \sqrt{3} \Gamma \gamma L) \approx 0.28 /(\gamma L),
$$

and the rms noise for distance measurements (in air) is

$$
\Delta z / \lambda=1 /(64 \sqrt{3} \Gamma \gamma L) \approx 0.025 /(\gamma L) .
$$

Typical values of the phase noise resulting from the quantization error are reported in Table 1. They are usually smaller than the one resulting from the shot noise. The most effective means of reducing the effect of the quantization error is to increase the number of bits used to sample the intensity range or to accumulate several measurements.

\section{ABSOLUTE ACCURACY}

We propose in this section to evaluate the absolute accuracy of the phase measurements. We consider the influence of deviation of the sinusoidal modulation parameters from their optimum values $\psi_{0}=2.45$ and $\theta_{0}=0.98$. Because of this deviation, an error $\delta$ appears in the calculation of the phase $\phi$ using Eq. (27). As the actual phase verifies Eq. (8), we have

$$
\tan (\phi+\delta)=\frac{\Sigma_{s}}{\Sigma_{c}}=\frac{\Gamma_{s}}{\Gamma_{c}} \tan \phi .
$$

Using a Taylor development at the second order, we can write

$$
\tan (\phi+\delta) \approx \tan \phi+\delta\left(1+\tan ^{2} \phi\right) .
$$

From Eqs. (38) and (39) we obtain an expression of the phase error as

$$
\delta=\frac{\rho-1}{2} \sin (2 \phi),
$$

where $\rho=\Gamma_{s} / \Gamma_{c}$ can be calculated by using Eq. (7). The error $\delta$ is zero when $\rho=1$, which is the case for $\psi=\psi_{0}$ and $\theta=\theta_{0}$. When $\psi$ and $\theta$ deviate from $\psi_{0}$ and $\theta_{0}$, an error appears, depending on the value of the phase $\phi$. An average value of the error (independent of $\phi$ ) can be obtained considering phase values uniformly distributed between 0 and $2 \pi$ through the phase map: 
Table 2. Absolute Accuracy of Phase Measurements as a Function of the Error on the PhaseModulation Parameters $\left(\psi=\psi_{0}+\Delta \psi, \theta=\theta_{0}+\Delta \theta\right.$, with $\psi_{0}=2.45$ and $\theta_{0}=0.96$ )

\begin{tabular}{cccc}
\hline $\begin{array}{c}\text { Error on } \\
\psi(\text { rad }) \\
|\Delta \psi|\end{array}$ & $\begin{array}{c}\text { Error on } \\
\theta \text { (rad) } \\
|\Delta \theta|\end{array}$ & $\begin{array}{c}\text { Average error } \\
\tilde{\delta} \text { (rad) } \\
\text { [from Eq. (44)] }\end{array}$ & $\begin{array}{c}\text { Accuracy } \Delta z \\
\text { [from (Eq. 45)] }\end{array}$ \\
\hline 0.02 & 0.01 & 0.01 & $\lambda / 830$ \\
0.04 & 0.02 & 0.03 & $N / 420$ \\
0.1 & 0.05 & 0.07 & $\lambda / 170$ \\
\hline
\end{tabular}

$$
\tilde{\delta}=\left[\frac{1}{2 \pi} \int_{0}^{2 \pi} \delta^{2}(\phi) \mathrm{d} \phi\right]^{1 / 2}=\frac{|\rho-1|}{2 \sqrt{2}} .
$$

Developing $\rho$ at the first order with respect to $\psi$ and $\theta$, we obtain

$$
\widetilde{\delta}=|0.30 \Delta \psi-0.71 \Delta \theta|
$$

where

$$
\psi=\psi_{0}+\Delta \psi, \quad \theta=\theta_{0}+\Delta \theta .
$$

Finally, an approximate upper bound of the phase error is given by

$$
\begin{gathered}
\tilde{\delta} \leqslant 0.3|\Delta \psi|+0.7|\Delta \theta|, \\
\Delta z / \lambda \leqslant 0.03|\Delta \psi|+0.06|\Delta \theta| .
\end{gathered}
$$

Typical values of the average phase error are reported in Table 2. For a wavelength in the visible region of the electromagnetic spectrum, the distance-measurement accuracy is of the order of $1 \mathrm{~nm}$ (as we shall see experimentally in Section 7).

\section{COMPARISON WITH LINEAR PHASE SHIFTING}

Phase-shifting interferometry that uses the integratingbucket technique is usually performed with a linear phase shift in a sawtoothlike manner. ${ }^{1,16-18}$ We propose to compare this technique to the one studied in this paper that consists of sinusoidal phase modulation with four integrating buckets. For the comparison to be pertinent, we consider a method of linear phase shifting by using the same number of buckets as described in Ref. 18. In this method, the phase is shifted linearly in a sawtoothlike manner with a total amplitude of $2 \pi \approx 6.2$, and four integrating buckets are recorded per period. The amplitude of the phase shift is 1.3 times higher than the optimal total amplitude of the sinusoidal phase modulation (equal to $2 \psi_{0}=4.9$ ). The accuracy of the phase calculation depends on the accuracy of the adjustment of the phase-modulation amplitude, which is similar for both methods. The influence of the additive noise was studied in Ref. 18. The resulting noise in the calculated phase map has a rms value given by

$$
\sqrt{\left\langle\varepsilon^{2}\right\rangle}=\pi /(4 \gamma \sqrt{N}),
$$

where $N$ is the average number of photoelectrons detected by each elementary detector during each bucket. Equation (46) is similar to the one calculated for the tech- nique studied in this paper [see Eq. (31)]. The noise is $\pi / 4 \approx 0.8$ times less than the one obtained with sinusoidal phase modulation. Linear and sinusoidal-phase modulations with four integrating buckets thus yield to similar performances. The advantage of sinusoidal phase modulation is that much higher modulation frequency is possible, especially when the modulation is generated by mechanical displacements. Indeed, a sawtoothlike oscillation becomes distorted at high frequency, whereas a sinusoidal oscillation remains steady. Moreover some phase modulators, such as a photoelastic birefringence modulator, naturally produce a sinusoidal phase modulation, which can be as high as several tens of $\mathrm{kHz}$, as we shall see in the next section.

\section{EXPERIMENTAL APPLICATIONS}

Phase modulation in an interferometer can be induced by many methods. ${ }^{1-4}$ Mechanical movements can be applied, such as moving a mirror or tilting a glass plate. An acousto-optic laser or a Zeeman laser can also be used. In interferometers with polarized beams, the modulation can be achieved by rotating a half-wave plate or a polarizer with an electro-optic modulator (Pockels effect), a magneto-optic modulator (Faraday effect), or a photoelastic birefringence modulator. We have applied the phasemeasurement technique studied in this paper to a homebuilt Linnik interference microscope ${ }^{23,24}$ by using two different methods to achieve sinusoidal phase modulation. We first present a method based on mechanical oscillation at $50 \mathrm{~Hz}$ induced by a PZT. Another method is demonstrated at much higher frequency using a photoelastic modulator operating at $50 \mathrm{kHz}$.

\section{A. Phase Modulation through Use of Mechanical Oscillation in an Interference Microscope}

Phase-shifting interferometry is usually based on the displacement of a mirror pushed by a PZT, step by step or linearly. We have built a Linnik interference microscope using a PZT to sinusoidally move the reference mirror at $50 \mathrm{~Hz}$, thus creating a sinusoidal phase shift in the interferometer. The experimental setup is depicted in Fig. 3. A CCD camera $(256 \times 256$ pixels, 8 bits $)$, operating at 200 $\mathrm{Hz}$, integrates the time-varying intensity on each pixel in

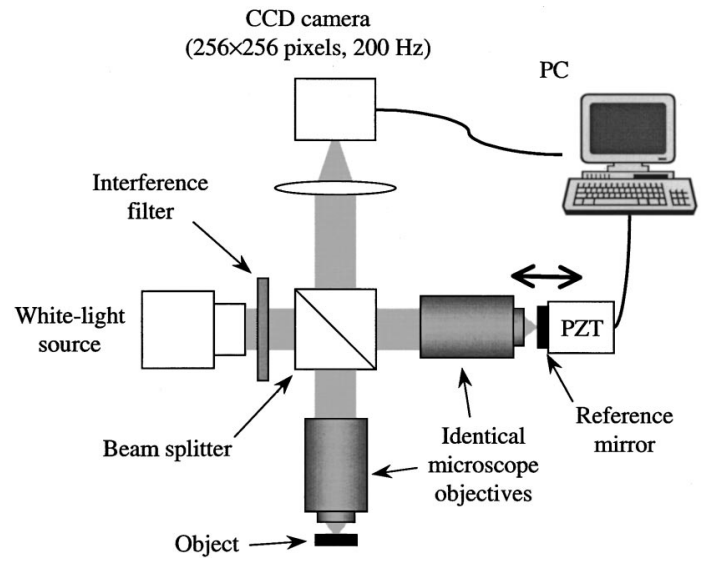

Fig. 3. Sinusoidally phase-modulated Linnik-type microscope using a PZT to make the reference mirror oscillate. 


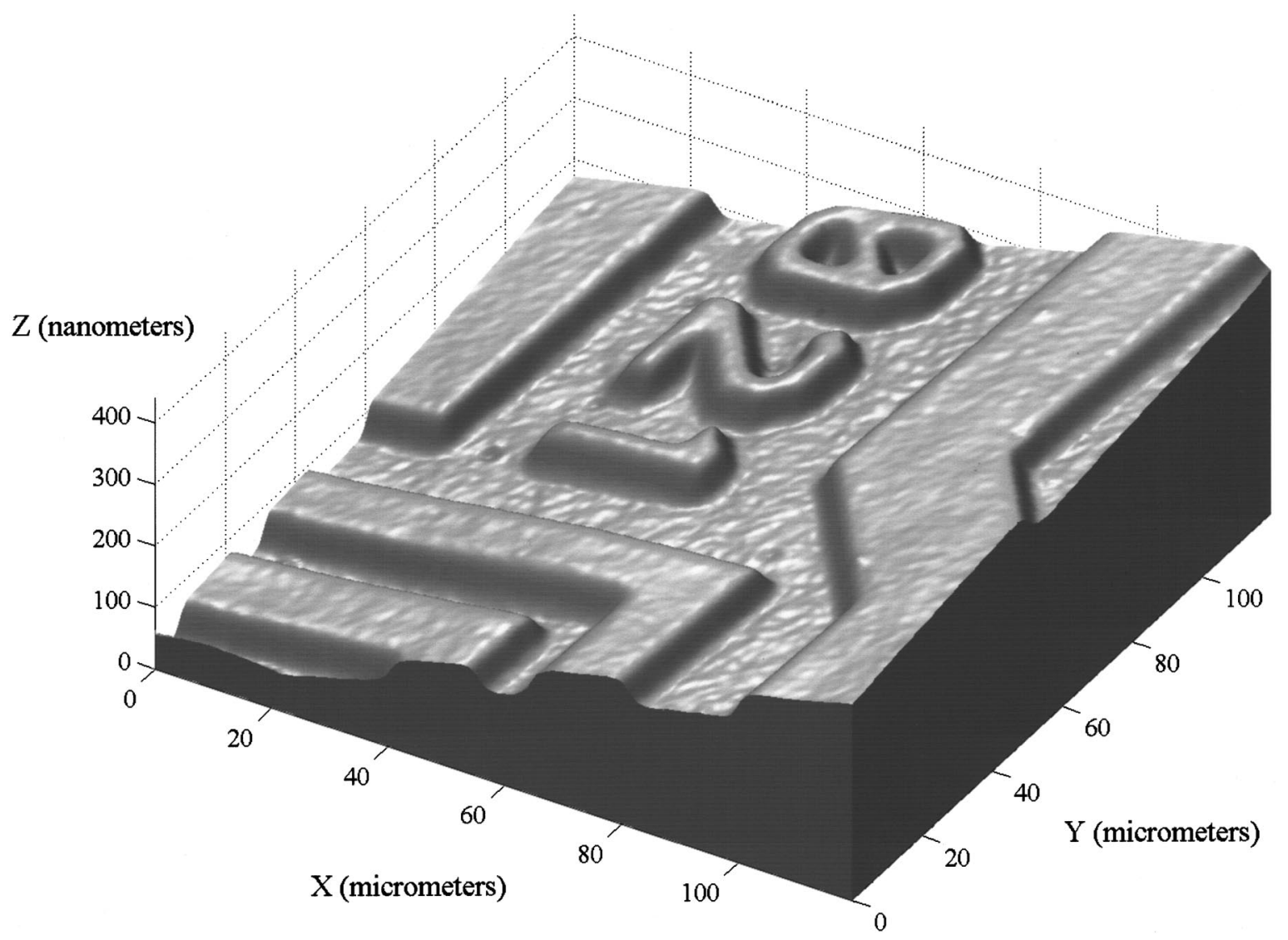

Fig. 4. Topographic image of details on a mask for integrated circuit manufacturing, obtained with our Linnik-type microscope.

parallel during the four quarters of the modulation period successively. Each series of four frames is used to calculate [using Eq. (27)] and produce a phase image in real time. The PZT oscillation is controlled by an amplifierposition servo controller by using an internal sine function at $50 \mathrm{~Hz}$ with adjustable amplitude to adjust the parameter $\psi$. The CCD camera is triggered with another power generator delivering a transistor-transistor logic (TTL) signal at $200 \mathrm{~Hz}$. The two generators are synchronized with an adjustable phase to adjust the parameter $\theta$. The experimental procedure to adjust $\psi$ and $\theta$ to their optimum values is the following: We take a mirror as the object and form a few interference fringes on its surface. Instead of a phase image, we produce in real time an image $\Xi$ calculated as $\Xi=\Sigma_{s}{ }^{2}+\Sigma_{c}{ }^{2}$. The parameter $\theta$ is adjusted so that the image $\Xi$ becomes zero. According to Eqs. (6) and (7), this happens when $\theta=0$. We estimated that this adjustment can be achieved with an accuracy of about $|\Delta \theta|=0.01 \mathrm{rad}$. The parameter $\theta$ is then set to its optimum value by adding $56^{\circ}(0.96 \mathrm{rad})$. The fluctuations of $\theta$ around this value are very weak (of the order of $10^{-5} \mathrm{rad}$ ). The image $\Xi$ exhibits fringes again. The parameter $\psi$ is then adjusted so that the fringes in the image $\Xi$ disappear, which means that $\Sigma_{s}{ }^{2}+\Sigma_{c}{ }^{2}$ no longer depends on the optical phase $\phi$. According to Eq. (6), this occurs when $\Gamma_{s}=\Gamma_{c}$. When the parameter $\theta$ is equal to 0.96 , the condition $\Gamma_{s}=\Gamma_{c}$ is verified for $\psi=2.45$ (see Fig. 2). Experimentally, we measured that this adjustment can be achieved with an accuracy of about $|\Delta \psi|=0.01 \mathrm{rad}$. According to the study presented in section 5 [see Eq. (45) and Table 2], the accuracy for height measurements is estimated to be $\sim \lambda / 800$. The microscope uses Köhler illumination with a dc-supplied quartz tungsten halogen lamp. Interference filters can be placed behind the source to reduce the spectral bandwidth to a few nanometers (for quasi-monochromatic illumination) and center the mean wavelength as desired. With a green filter $(\lambda=545 \mathrm{~nm})$, the peak-to-peak amplitude of the sinusoidal PZT displacement is $212 \mathrm{~nm}$. Phase images produced by the microscope can be accumulated and smoothed to reduce the noise if necessary. As the phase is related to the height difference between the surface of the object and the surface of the reference mirror, three-dimensional images of reliefs can be obtained. ${ }^{25}$ The calculations are performed with a $800-\mathrm{MHz}$ Pentium III computer using a Visual $\mathrm{C}++$ compiled interface to control the image acquisition, calculate the phase maps, and unwrap them with the Goldstein algorithm. ${ }^{26}$ The final three-dimensional representations are produced by Matlab. A topographic image of a $120 \mu \mathrm{m}^{2}$ region on the surface of a mask for integrated circuit manufacturing is shown in Fig. 4. This image was obtained with an acquisition time of $0.2 \mathrm{~s}$ at the wavelength of $545 \mathrm{~nm}$.

\section{B. Phase Modulation Through Use of Birefringence Modulation in an Interference Microscope}

We have also adapted the phase-measurement scheme described in this paper to our Linnik-type interference microscope using a photoelastic birefringence modulator to achieve sinusoidal phase modulation. ${ }^{27}$ A schematic of 


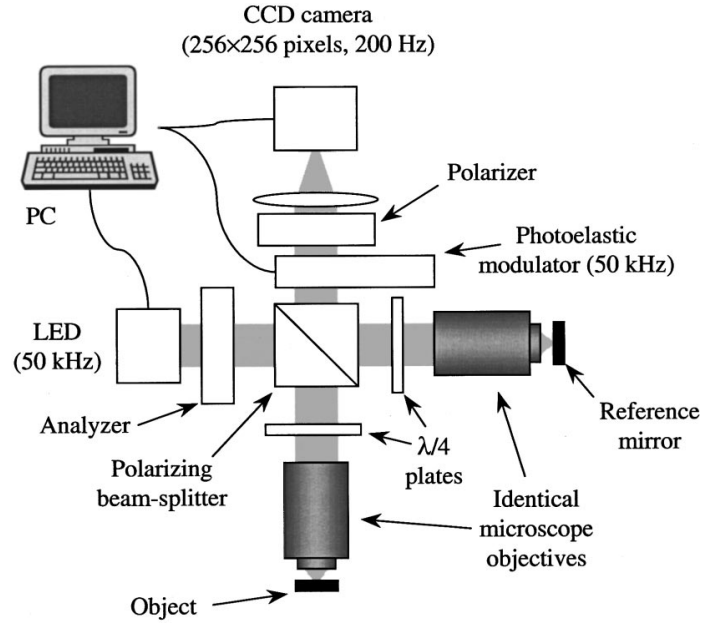

Fig. 5. Sinusoidally phase-modulated polarization Linnik-type microscope using a photoelastic birefringence modulator and stroboscopic illumination.

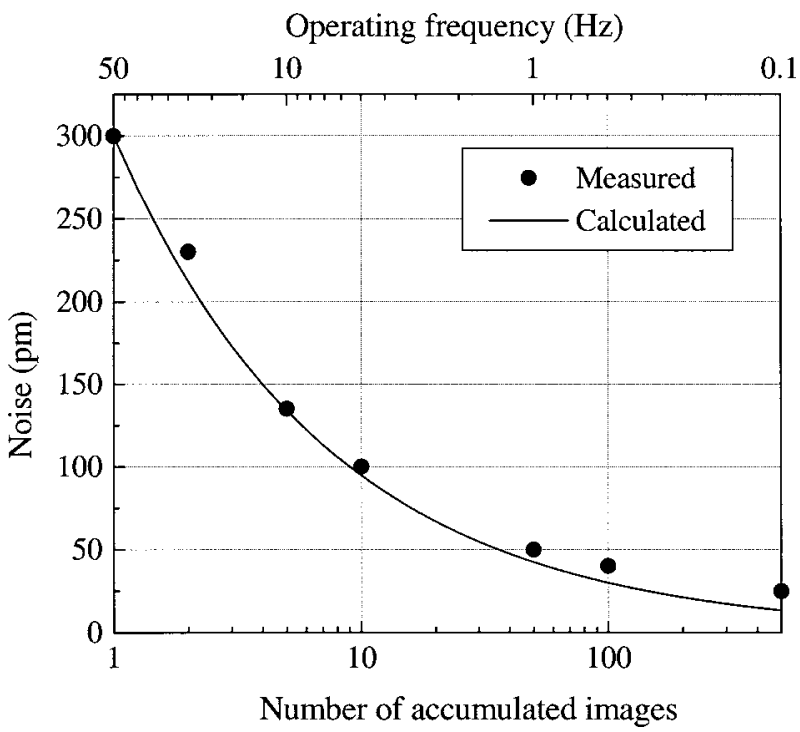

Fig. 6. Noise measurements. Comparison with theory assuming our system to be shot-noise limited $(N=100000, \gamma=0.7)$.

the experimental setup is represented in Fig. 5. The interference fringes are shifted sinusoidally at the frequency $f=50 \mathrm{kHz}$ with the photoelastic modulator. Because the CCD camera is obviously not able to operate at $4 f=200 \mathrm{kHz}$, the integrating-bucket method cannot be performed directly with the camera as previously described. We use here stroboscopic illumination with a light-emitting diode (LED) at $840 \mathrm{~nm}$ that is set on during $1 / 4$ of the modulation period $T=1 / f$, then off during $3 / 4$ of $\mathrm{T}$, and so on. The camera integrates the signal over 250 modulation periods $(50 \mathrm{kHz} / 200 \mathrm{~Hz}=250)$. This leads to a first frame, which is put in memory. Then the stroboscopic illumination is shifted by $1 / 4$ of $T$ to acquire a second frame, and so on. Four frames with different interference fringe patterns are thus recorded successively by the CCD camera and are stored in memory. During the acquisition of the next set of four frames, calculations are performed with the previous four frames to produce a phase image in real time. Specific electronics was developed to control the synchronization of the sinusoidal phase modulation at $50 \mathrm{kHz}$ with both the periodically phase-shifted stroboscopic illumination at $50 \mathrm{kHz}$ and the camera at $200 \mathrm{~Hz}$. Several phase images can be accumulated and smoothed to reduce the noise if necessary. The peak-to-peak amplitude of the sinusoidal birefringence modulation induced by the photoelastic modulator is given by

$$
\Delta n=\frac{\psi_{0} \lambda}{2 \pi e}=0.39 \frac{\lambda}{e} .
$$

Given that the thickness of the modulator rod is $e=20 \mathrm{~mm}$ and the wavelength $\lambda=840 \mathrm{~nm}$, the amplitude of the birefringence is $\Delta n=1.64 \times 10^{-5}$. This birefringence value is easily obtained with low applied power.

\section{Noise Measurements}

We have measured the noise present in the phase images delivered by the Linnik interference microscope described previously. The procedure was to take two phase images successively and calculate their difference. The standard deviation of the difference, for all the pixels, gives the rms total noise. At the maximal phase-image acquisition frequency of $50 \mathrm{~Hz}$, the noise was measured to be $\sim 4.5 \times 10^{-3} \mathrm{rad}$ (300 pm with $\lambda=840 \mathrm{~nm}$ ). As expected, the accumulation of images reduces the noise. At $1 \mathrm{~Hz}$ (50 phase images accumulated), a noise of $\sim 7.5 \times 10^{-4} \mathrm{rad}$ (50 pm with $\lambda=840 \mathrm{~nm}$ ) was reached. The measurements were compared with theory, assuming the system to be purely shot-noise limited [see Eqs. (31) and (33)]. The results are presented in Fig. 6. Considering the CCD camera has an average well-charge storage of 100,000 per pixel and the fringe contrast $\gamma$ is equal to 0.7 , we found good agreement with experiments for exposure times less than one second typically. For longer exposure times, the experimental results deviate from the shot-noise limit because of diverse instability sources (mechanical vibrations, thermal drifts, etc.) that begin to take place. Smoothing the images with a Sobel filter can reduce the noise by a factor two without significant loss of lateral resolution.

\section{Other Applications}

Several other interferometer systems were developed in the laboratory, all based on the phase measurement scheme described previously: a photoelastic birefringence modulator to achieve sinusoidal phase modulation and a CCD camera to integrate the signal in parallel for a large number of pixels. Stroboscopic illumination with a light-emitting diode transposes the high modulation frequency imposed by the mechanical resonance of the photoelastic rod to a frequency compatible with the readout frequency of the CCD camera. A Nomarski microscope ${ }^{28}$ was developed on this principle. In addition to microscopes devoted to the study of objects at high spatial frequencies, other instruments were developed for applications at low spatial frequencies. A system to measure the birefringence of large optics with sensitivity of the order of $10^{-4}$ was proposed recently. ${ }^{29}$ A lateral shear interferometer ${ }^{30}$ is being constructed currently. 


\section{CONCLUSION}

We studied a method to get phase images from doublebeam interferometers using the integrating-bucket technique with sinusoidal phase modulation. Optimum values for the amplitude and phase of the sinusoidal phase modulation were found to minimize the effect of the additive noise. The technique was applied on several interferometer systems to produce images in real time at a rate of several hertz. The phase-measurement accuracy was estimated to be of the order of $10^{-2} \mathrm{rad}$. A minimum noise of $\sim 5.10^{-4}$ rad was measured. The method is currently under study to be applied on interferometers using broad-bandwidth illumination, such as white-light interferometers. ${ }^{31,32}$

\section{ACKNOWLEDGMENT}

The author is grateful for fruitful discussions with A. C. Boccara during the preparation of this manuscript.

The author's e-mail address is dubois@optique.espci.fr.

\section{REFERENCES}

1. K. Creath, "Phase-measurements interferometry techniques," in Progress in Optics, E. Wolf, ed. (Elsevier Science, New York, 1988), Vol. 26, pp. 349-393.

2. J. Schwider, "Advanced evaluation techniques in interferometry," in Progress in Optics, E. Wolf, ed. (Elsevier Science, New York, 1990), Vol. 28, pp. 271-359.

3. J. E. Greivenkamp and J. H. Bruning, "Phase shifting interferometers," in Optical Shop Testing, D. Malacara, ed. (Wiley, New York, 1991), Chap. 14, pp. 501-598.

4. R. S. Sirohi and M. P. Kothiyal, "Heterodyne and phase shifting interferometry," in Optical Components, Systems, and Measurement Techniques, R. S. Sirohi and M. P. Kothiyal, eds. (Marcel Dekker, New York, 1991), pp. 219 246.

5. D. W. Phillion, "General methods for generating phaseshifting interferometry algorithms," Appl. Opt. 36, 8098 8115 (1997)

6. P. Carré, "Installation et utilisation du comparateur photoélectrique et interférentiel du bureau international des poids et mesures," Metrologia 2, 13-23 (1966).

7. P. Hariharan, B. F. Oreb, and T. Eiju, "Digital phaseshifting interferometry: a simple error-compensating phase calculating algorithm," Appl. Opt. 26, 2504-2506 (1987).

8. K. G. Larkin and B. F. Oreb, "Design and assessment of symmetrical phase-shifting algorithms," J. Opt. Soc. Am. A 9, 1740-1748 (1992).

9. Y. Surrel, "Phase stepping: a new self-calibrating algorithm," Appl. Opt. 39, 3598-3600 (1993).

10. J. Schwider, O. Falkenstorfer, H. Schreiber, A. Zoller, and N. Streibl, "New compensating four-phase algorithm for phase-shift interferometry," Opt. Eng. 32, 1883-1885 (1993).
11. B. Zhao and Y. Surrel, "Phase shifting: six-sample selfcalibrating algorithm insensitive to the second harmonic in the fringe signal," Opt. Eng. 34, 2821-2822 (1995).

12. P. de Groot, "Derivation of algorithms for phase-shifting interferometry using the concept of a data-sampling window," Appl. Opt. 34, 4723-4730 (1995).

13. J. Schmit and K. Creath, "Extended averaging technique for derivation of error compensating algorithms in phaseshifting interferometry," Appl. Opt. 34, 3610-3619 (1995).

14. Y. Surrel, "Design of algorithms for phase measurements by the use of phase stepping," Appl. Opt. 35, 51-60 (1996).

15. K. G. Larkin, "Efficient nonlinear algorithm for envelope detection in white light interferometry," J. Opt. Soc. Am. A 13, 832-843 (1996).

16. K. D. Stumpf, "Real-time interferometer," Opt. Eng. 18, 648-653 (1979).

17. B. Bhushan, J. C. Wyant, and C. L. Koliopoulos, "Measurements of surfaces topography of magnetic tapes by Mirau interferometry," Appl. Opt. 24, 1489-1497 (1985).

18. J. C. Wyant, "Use of an ac heterodyne lateral shear interferometer with real-time wavefront correction systems," Appl. Opt. 14, 2622-2626 (1975).

19. O. Sasaki and H. Okazaki, "Sinusoidal phase modulating interferometry for surface profile measurement," Appl. Opt. 25, 3137-3140 (1986).

20. O. Sasaki and H. Okazaki, "Analysis of measurement accuracy in sinusoidal phase modulating interferometry," Appl. Opt. 25, 3152-3158 (1986).

21. O. Sasaki, H. Okazaki, and M. Sakai, "Sinusoidal phase modulating interferometer using the integrating-bucket method," Appl. Opt. 26, 1089-1093 (1987).

22. C. P. Brophy, "Effect of intensity error correlation on the computed phase of phase-shifting interferometry," J. Opt. Soc. Am. A 7, 537-541 (1990).

23. M. Davidson, K. Kaufman, I. Mazor, and F. Cohen, "An application of interference microscopy to integrated circuit inspection and metrology," in Integrated Circuit Metrology, Inspection, and Process Control, K. M. Monahan, ed., Proc. SPIE 775, 233-247 (1987).

24. G. S. Kino and S. C. Chim, "Mirau correlation microscope," Appl. Opt. 29, 3775-3783 (1990).

25. A. Dubois, J. Selb, L. Vabre, and A. C. Boccara, "Phase measurements with wide-aperture interferometers," Appl. Opt. 39, 2326-2331 (1999).

26. D. C. Ghiglia and M. D. Pritt, Two-Dimensional Phase Unwrapping, Theory, Algorithms, and Software (Wiley, New York, 1998).

27. A. Dubois, A. C. Boccara, and M. Lebec, "Real-time reflectivity and topography imagery of depth-resolved microscopic surfaces," Opt. Lett. 24, 309-311 (1999).

28. P. Gleyzes, A. C. Boccara, and H. Saint-Jalmes, "Multichannel Nomarski microscope with polarization modulation: performance and applications," Opt. Lett. 22, 1529-1531 (1997).

29. G. de Villèle and V. Loriette, "Birefringence imaging with imperfect benches: application to large-scale birefringence measurements," Appl. Opt. 39, 3864-3874 (2000).

30. D. Malacara, "Common-path interferometers," in Optical Shop Testing, 2nd ed., J. W. Goodman, ed. (Wiley, New York, 1992), pp. 106-108.

31. P. A. Flourney, R. W. McClure, and G. Wyntjes, "White-light interferometric thickness gauge," Appl. Opt. 11, 1907-1915 (1972).

32. P. J. Caber, "Interferometric profiler for rough surfaces," Appl. Opt. 32, 3438-3441 (1993). 